\title{
ÉTUDE DU COMPORTEMENT DE SMOLTS DE SAUMON ATLANTIQUE (SALMO SALAR L.) AU NIVEAU DE LA PRISE D'EAU DE L'USINE HYDROÉLECTRIQUE DE POINTIS SUR LA GARONNE ET ESTIMATION DE LA DÉVALAISON AU NIVEAU DU BARRAGE DE RODĖRE.
}

\section{O. CROZE et M. LARINIER}

\begin{abstract}
CSP-CEMAGREF-GHAAPPE, Institut de Mécanique des Fluides, Avenue du Professeur Camille Soula, 31400 Toulouse, France.
\end{abstract}

\section{RÉSUMÉ}

Une expérimentation a été menée en 1998 au niveau de la prise d'eau de l'usine hydroélectrique de Pointis, sur la Garonne, de manière à déterminer, grâce à la technique de radio-pistage, le meilleur emplacement d'un futur dispositif de dévalaison. Le suivi des déplacements de 28 smolts radiomarqués a mis en évidence que, dans des conditions hydrauliques peu marquées (vitesses de courant $<0,5-0,6 \mathrm{~m} / \mathrm{s}$ ), les poissons peuvent stationner des temps conséquents (valeur médiane supérieure à 1 jour) en amont immédiat de l'usine et ce, malgré un espacement de $5 \mathrm{~cm}$ entre les barreaux des grilles de prise d'eau. Durant leur temps de présence dans le canal d'amenée, les poissons explorent la totalité de la prise d'eau en amont des grilles.

Une lampe placée en rive gauche, à l'amont immédiat des grilles de prise d'eau, concentre les poissons à proximité de la zone éclairée.

Une estimation des dévalaisons au niveau du barrage de Rodère a aussi été réalisée. Ce barrage, situé en aval de l'aménagement de Pointis, permet d'alimenter en eau le canal d'amenée de l'usine hydroélectrique de Camon. La proportion de smolts dévalant par le barrage est liée à l'importance des déversements au barrage. II faut néanmoins attendre des débits déversés de 40 à $50 \%$ du débit total de la Garonne pour voir dévaler par le barrage $70 \%$ des poissons.

Les délais nécessaires aux smolts pour effectuer les $5,4 \mathrm{~km}$ séparant l'amont de Pointis de l'usine de Camon apparaissent très variables ( 1 heure 17 minutes à 12 jours ; médiane $>1$ jour) et dépendent du débit du cours d'eau. Les vitesses de dévalaison dans les canaux d'amenée de Pointis $(1,9$ à $4,2 \mathrm{~km} / \mathrm{h})$ et de Camon $(2,5$ à $7 \mathrm{~km} / \mathrm{h})$ sont positivement corrélées aux vitesses de l'écoulement.

Mots-clés : dévalaison, smolt, saumon atlantique, aménagement hydroélectrique, barrage, radio-pistage, comportement, lumière. 


\title{
A STUDY OF ATLANTIC SALMON (SALMO SALAR L.) SMOLT BEHAVIOUR AT THE POINTIS HYDROELECTRIC POWERHOUSE WATER INTAKE ON THE GARONNE RIVER AND AN ESTIMATE OF DOWSTREAM MIGRATION OVER THE RODĖRE DAM.
}

\begin{abstract}
An experiment was conducted in 1998 at the Pointis water intake, on the Garonn: River, to define location for a future downstream bypass for Atlantic salmon smolts. The study of the movements of 28 radiotagged smolts revealed that under low hydraulic: conditions (flow velocities $<0.5-0.6 \mathrm{~m} / \mathrm{s}$ ), the smolts maintain their position for a significan time (median time greater than one day) in the intake canal, despite the large spacing $(5 \mathrm{~cm})$ between the bars of the trashrack. During this time, they explore the whole area of the intake canal above the trashrack.
\end{abstract}

A light placed on the left bank just upstream from the trashrack concentrated the fish on the same bank as the lamp, in the vicinity of the lighted area.

The proportion of fish passing downstream from the Rodère dam itself was estimated. This dam, located downstream from the Pointis plant, supplies the Camon hydroelectric powerhouse. It appeared that the proportions of smolts passing through the Rodère dam were related to the ratio of discharge at the dam to the total Garonne flow : $70 \%$ of the smolts passed by when the discharge at the dam corresponded to $40-50 \%$ of the total Garonne flow.

The time taken by radiotagged smolts to go from the Pointis plant to the Camon powerhouse $(5.4 \mathrm{~km}$ ) varied from 1 hour 17 minutes to 12 days with a median value of more than one day, and depended on river discharges. The downstream displacements in the intake canals of Pointis $(1.9$ to $4.2 \mathrm{~km} / \mathrm{h})$ and Camon $(2.5$ to $7 \mathrm{~km} / \mathrm{h})$ were positively correlated with flow velocities.

Key-words : downstream migration, smolt, Atlantic salmon, hydroelectric power plant, dam, radio-tracking, behaviour, light.

\section{INTRODUCTION}

L'étude conduite en 1998 à l'usine hydroélectrique de Pointis sur la Garonne fait suite à la décision d'équiper ce site d'un dispositif de dévalaison, dans le cadre du plan de réouverture de la Garonne amont à la dévalaison des salmonidés grands migrateurs. Compte tenu des conditions hydrauliques peu marquées dans le canal d'amenée et des contraintes liées au site, il a paru préférable d'étudier le comportement des smolts à l'amont des grilles de la prise d'eau, de manière à déterminer l'emplacement du futur exutoire.

Parallèlement, il a semblé intéressant d'une part d'évaluer les vitesses de migration des smolts entre le site de Pointis et le premier aménagement situé à l'aval (Rodère-Camon), et d'autre part, de préciser comment évoluaient, en fonction des déversements au niveau du barrage de Rodère, les proportions respectives des individus transitant directement par le barrage et de ceux entraînés dans le canal d'amenée de l'usine de Camon. 


\section{MATÉRIEL ET MÉTHODES}

\section{Site d'expérimentation}

L'aménagement hydroélectrique de Pointis est situé dans le sud-ouest de la France, sur la Garonne, à $87 \mathrm{~km}$ de la source et $118 \mathrm{~km}$ en amont de Toulouse (Figure 1) soit environ $3 \mathrm{~km}$ en amont de l'aménagement de Camon décrit par CROZE, CHANSEAU et LARINIER (1999). Le bassin versant naturel au niveau de cet aménagement est voisin de $2100 \mathrm{~km}^{2}$. Le régime des eaux est de type pluvio-nival. Le module interannuel est voisin de $52 \mathrm{~m}^{3} / \mathrm{s}$, les débits moyens mensuels lors de la période de dévalaison étant de $50 \mathrm{~m}^{3} / \mathrm{s}$ en mars, $73 \mathrm{~m}^{3} / \mathrm{s}$ en avril et $97 \mathrm{~m}^{3} / \mathrm{s}$ en mai.
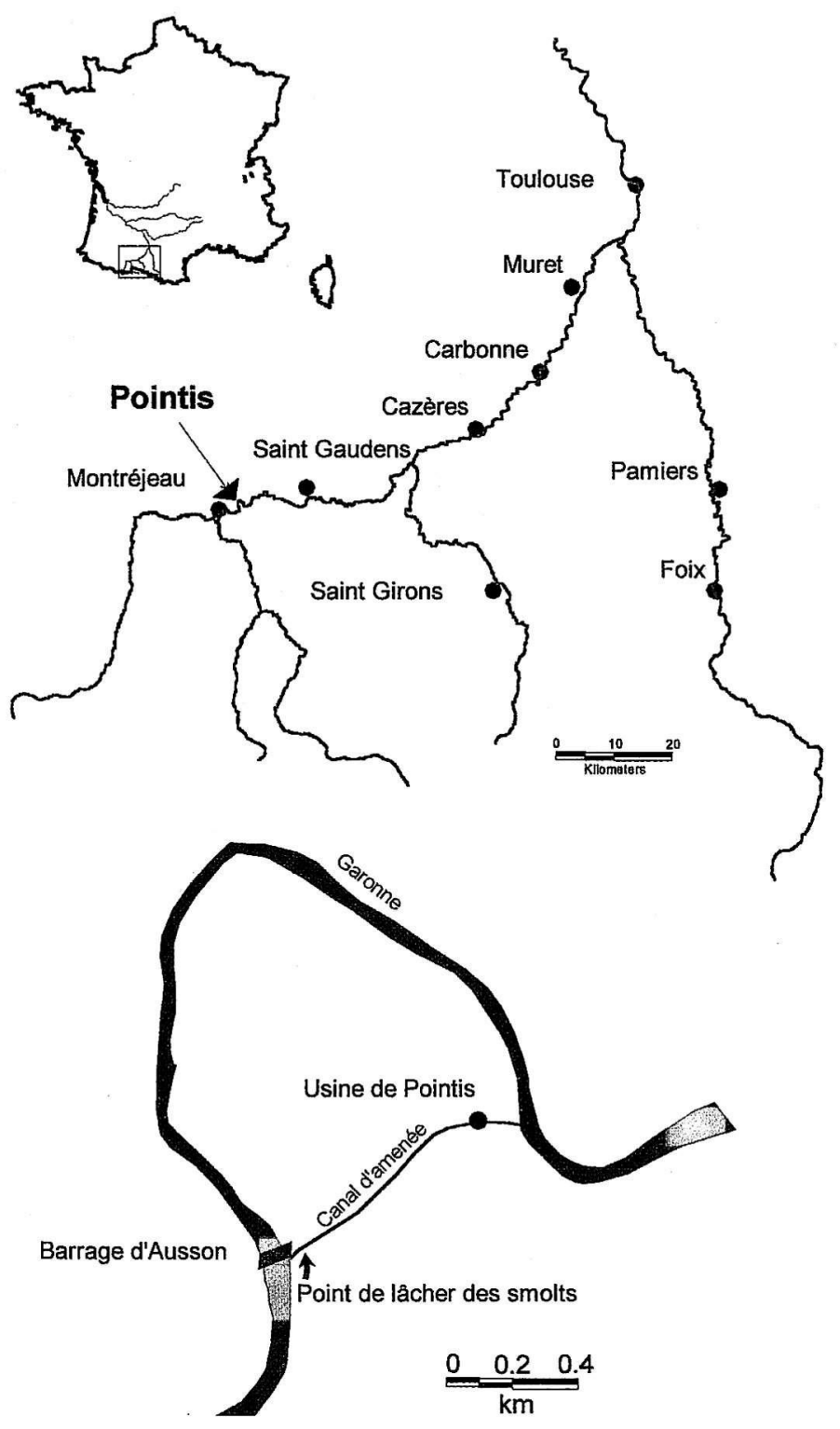

Figure 1

Situation géographique et vue générale de l'aménagement hydroélectrique de Pointis.

\section{Figure 1}

Geographical situation and general view of Pointis hydroelectric plant. 
L'aménagement de Pointis comprend un barrage mobile (Ausson) de $66 \mathrm{~m} d$ ? largeur et d'une hauteur de 5,50 m qui permet d'alimenter, par un canal de $700 \mathrm{~m}$ de lonc, la centrale hydroélectrique de Pointis. Cette centrale (Figure $2 A$ ) est équipée de trois turbines de type hélice à quatre pales (puissance nominale : $2500 \mathrm{~kW}$ par hélice, chut: nette : $13 \mathrm{~m}$ ). Le débit maximum turbinable est voisin de $70 \mathrm{~m} 3 / \mathrm{s}$.

Le canal d'amenée a une largeur voisine de $20 \mathrm{~m}$ et une profondeur de $5,9 \mathrm{~m}$. La prise d'eau à l'usine mesure 21,5 m de largeur (Figures 2B et 3). Les grilles de protectior, d'une hauteur totale de $8,5 \mathrm{~m}$, sont constituées de barreaux rectangulaires $(1 \mathrm{~cm}$ par $8 \mathrm{~cm}$ ) présentant un espacement de $5 \mathrm{~cm}$.

Le canal de fuite de l'usine de Pointis, d'une longueur de $90 \mathrm{~m}$, rejoint la Garonn? un peu plus d'un kilomètre en amont du barrage de Rodère. Ce barrage, d'une hauteur de $6 \mathrm{~m}$, barre la Garonne pour alimenter en eau l'aménagement hydroélectrique de Camon (CROZE, CHANSEAU et LARINIER, 1999). Il est constitué de 3 vannes wagons de $20 \mathrm{~m}$ de largeur chacune (Figures 2C, 2D et 4).
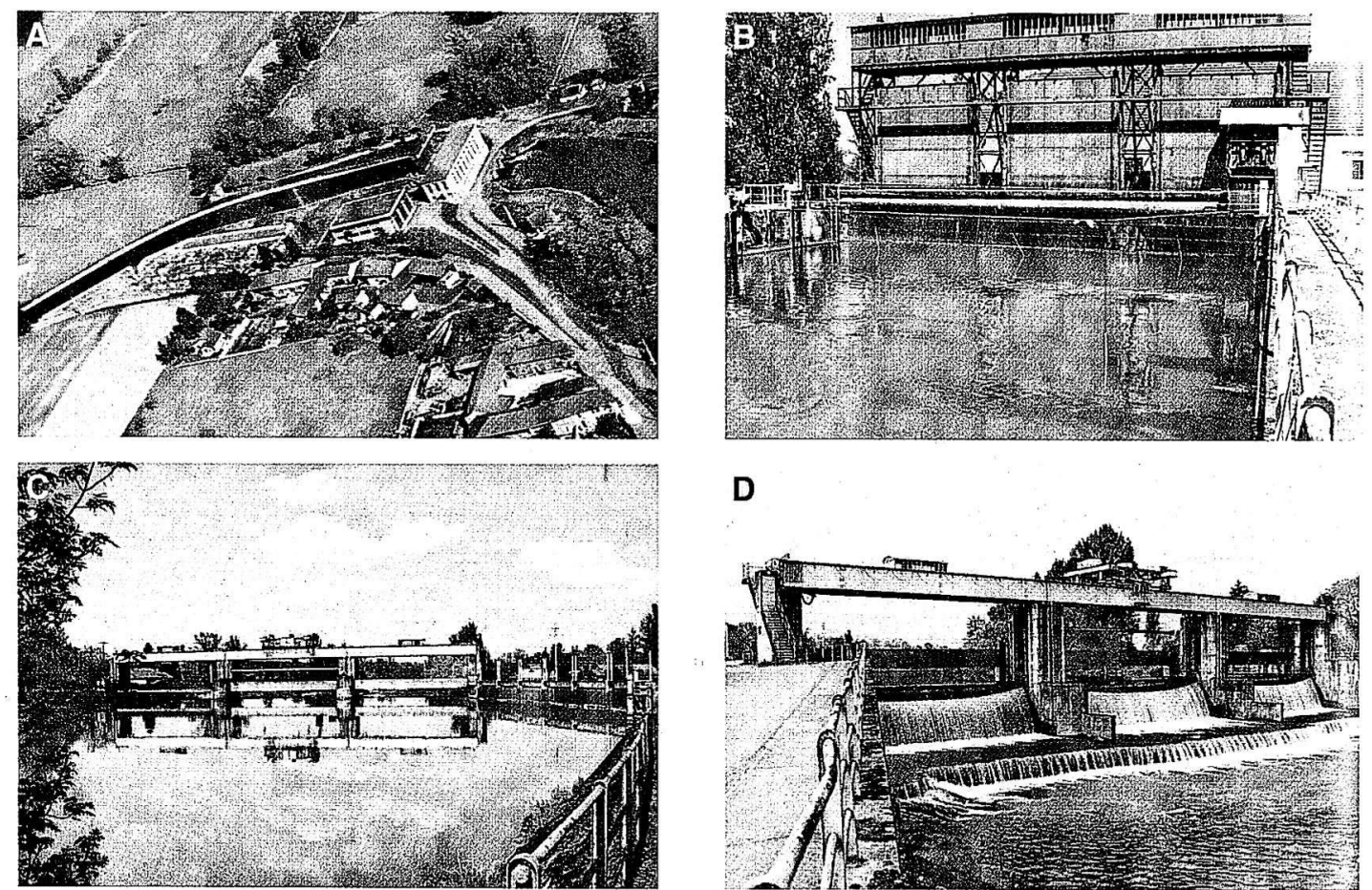

D

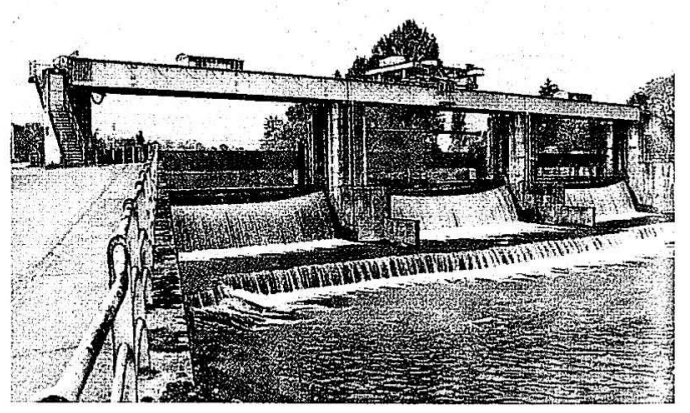

Figure 2A

Vue aérienne de l'usine hydroélectrique de Pointis.

Figure 2B

Vue amont de la prise d'eau de l'usine de Pointis.

Figure 2C

Vue amont du barrage de Rodère.

Figure 2D

Vue aval du barrage de Rodère.

Figure 2A

Aerial view of Pointis hydroelectric powerhouse.

Figure 2B

Upstream view of Pointis power intake.

Figure 2C

Upstream view of Rodère dam.

Figure 2D

Downstream view of Rodère dam. 


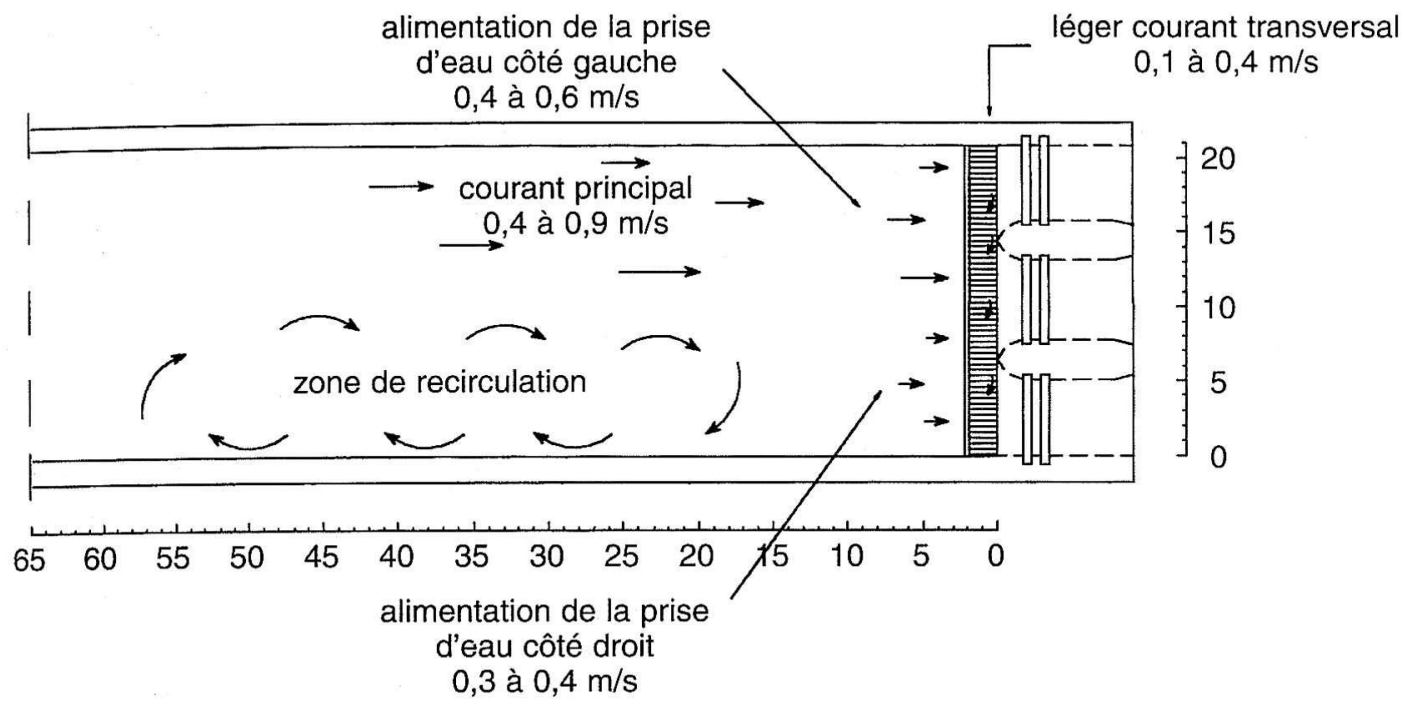

Figure 3

Vue générale de la prise d'eau de l'usine hydroélectrique de Pointis et hydrodynamique du canal d'amenée.

Figure 3

General view of Pointis hydroelectric power intake and velocity patterns in the forebay.

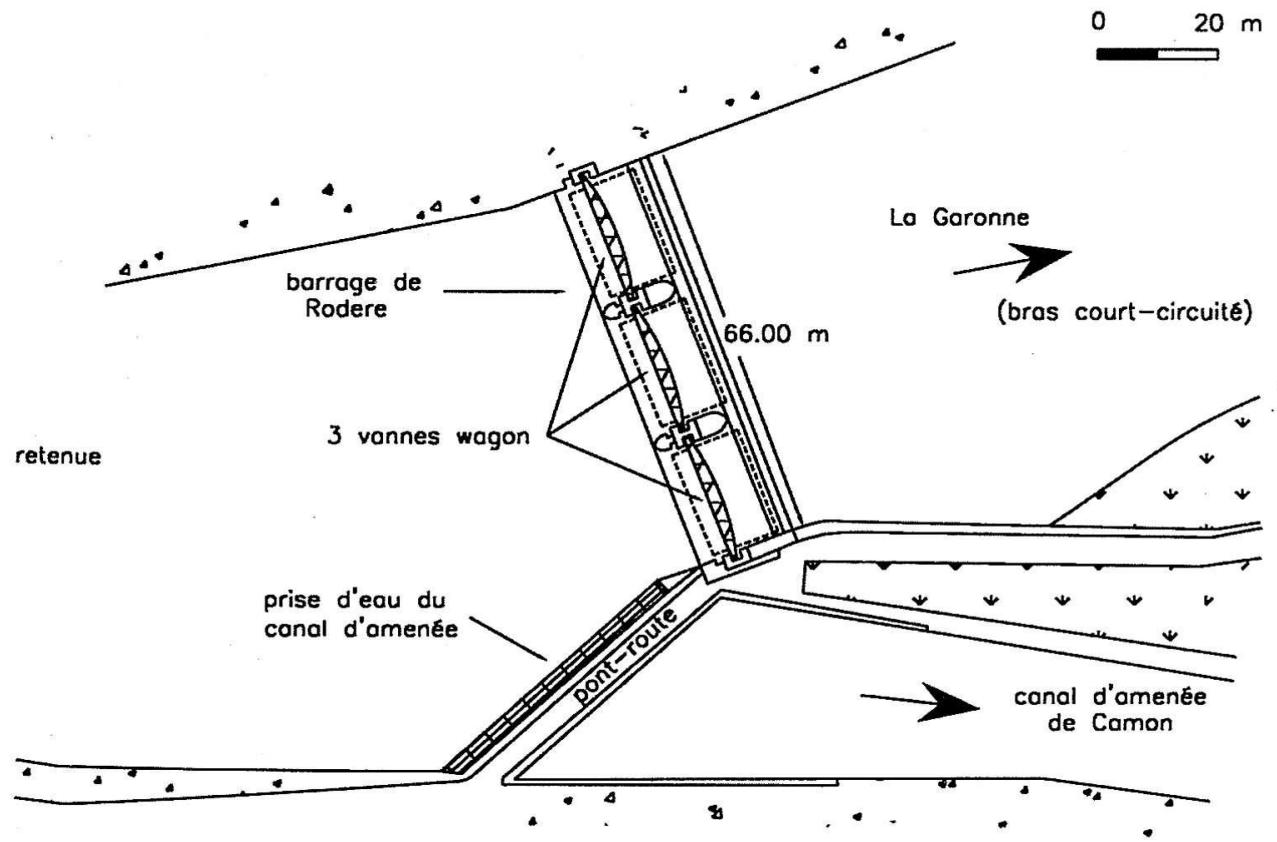

Figure 4

Vue générale du barrage de Rodère et de la prise d'eau du canal d'amenée de l'usine hydroélectrique de Camon.

\section{Figure 4}

General view of Rodère dam and Camon river intake. 


\section{Hydrodynamique en amont de la prise d'eau de l'usine}

L'alimentation de la prise d'eau se fait préférentiellement par la rive gauche. Les valeurs des vitesses de l'écoulement en surface, mesurées à l'aide de flotteurs, pour un débit dans le canal d'amenée proche de son maximum, sont comprises entre $0,4 \mathrm{~m} / \mathrm{s}$ e $0,9 \mathrm{~m} / \mathrm{s}$. En rive droite du canal d'amenée, une grande zone de recirculation s'étend d'une quinzaine de mètres à plus de $60 \mathrm{~m}$ en amont des grilles (Figure 3 ). En aval de cette zone les vitesses sont normales au plan de grille et restent modérées: les valeurs maximales de l'ordre de $0,6 \mathrm{~m} / \mathrm{s}$, sont observées du côté gauche. Ces vitesses sont nettement moins importantes du côté droit où elles ne dépassent pas 0,3-0,4 m/s.

Un léger courant transversal (vitesses inférieures à $0,4 \mathrm{~m} / \mathrm{s}$ ), dirigé de la rive gauche vers la rive droite, a été mis en évidence au droit des grilles de l'usine.

\section{Paramètres du milieu}

La température, le niveau d'eau en amont des grilles de la prise d'eau et la puissance fournie par les trois turbines de l'usine de Pointis ont fait l'objet d'enregistrements en continu pendant les expérimentations au moyen de centrales d'acquisition portables autonomes.

\section{Période d'étude}

L'expérimentation a débuté le 6 avril pour se terminer le 20 mai 1998. Elle s'inscrit dans la période naturelle de migration de dévalaison des smolts de saumon atlantique dans la partie méridionale de l'Europe (BAGLINIERE, 1976; BOUSQUET, 1979; METCALFE et THORPE, 1990).

\section{Comportement des smolts}

Le comportement de 28 smolts marqués à l'aide d'un émetteur a été étudié grâce à la technique de radio-pistage. Le matériel utilisé est identique à celui employé par CHANSEAU, LARINIER et TRAVADE (1999). Dix-neuf zones de réception ont été individualisées en amont de la prise d'eau de l'usine (Figure 5) : les déplacements des poissons radiomarqués ont été enregistrés en continu sur les 45 derniers mètres du canal d'amenée. Ces poissons, issus de pisciculture, ont été lâchés de nuit avec une vingtaine d'autres smolts à raison d'un ou deux smolts radiomarqués par lot. Les lâchers ont été effectués dans le canal d'amenée de Pointis, une trentaine de mètres en aval de la prise d'eau de ce canal, soit à environ $700 \mathrm{~m}$ en amont des grilles de l'usine hydroélectrique de Pointis.

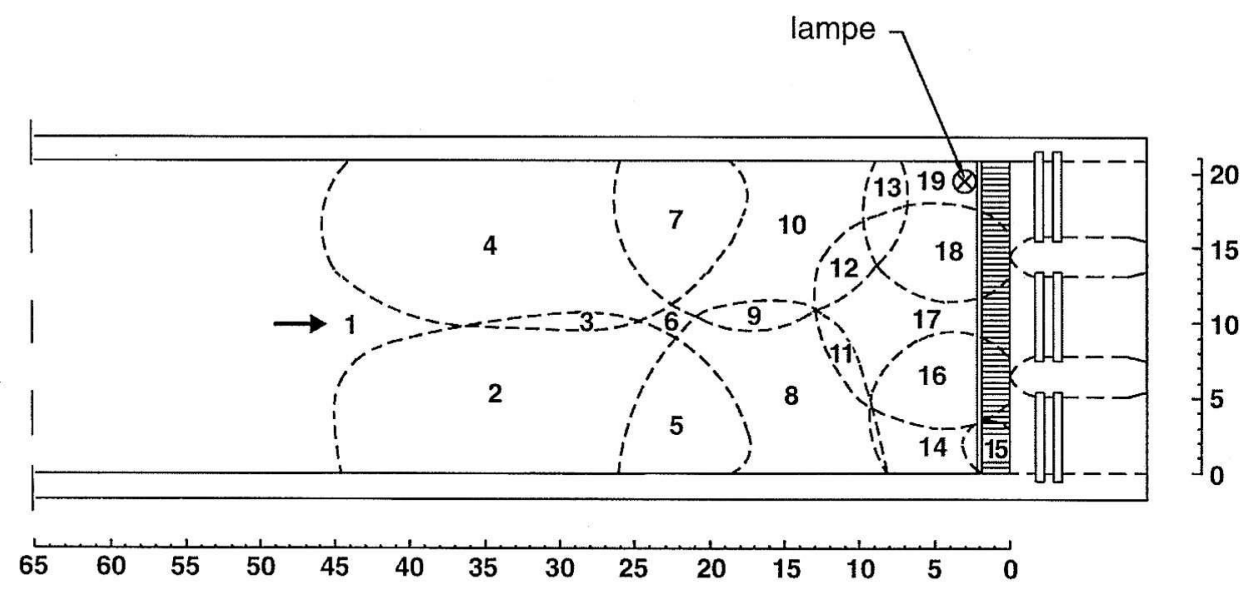

Figure 5

Zones de réception des radioémetteurs.

\section{Figure 5}

Radiotelemetry listening areas. 


\section{Influence de la lumière}

Afin de tester les effets d'une source lumineuse sur les déplacements des poissons, une lampe à vapeur de mercure d'une puissance de $80 \mathrm{~W}$ a été placée en rive gauche à $1,5 \mathrm{~m}$ de la berge et à $2,4 \mathrm{~m}$ en amont des grilles (Figure 5). Cet éclairage continu a fonctionné en moyenne une nuit sur deux sur l'ensemble de la campagne.

\section{Estimation des dévalaisons par le barrage de Rodère et temps de transit entre Pointis et Camon}

Le suivi des smolts radiomarqués après leur passage à l'aval de l'usine de Pointis a permis d'évaluer leur temps de transit jusqu'au barrage de Rodère et, pour certains individus, jusqu'au niveau de la prise d'eau de l'usine de Camon.

Les smolts lâchés avec les poissons équipés d'un émetteur ont été marqués à l'aide d'un transpondeur introduit dans la cavité générale, légèrement au-dessus de la ligne médio-ventrale, à l'arrière des pectorales. Ces «PIT tags » $(11 \mathrm{~mm}$ de long pour $2 \mathrm{~mm}$ de diamètre) possèdent un code alphanumérique individuel. Une plaque de détection (30 $\mathrm{cm} \times 97 \mathrm{~cm}$ ) a été installée au niveau de l'exutoire de dévalaison de l'usine hydroélectrique de Camon et a permis d'enregistrer la date et l'heure des passages des poissons marqués.

Dix-neuf lots d'une vingtaine d'individus chacun ont été lâchés en amont de l'usine de Pointis. Le nombre de smolts dévalant au niveau du barrage de Rodère a été estimé en fonction du nombre d'individus capturés à l'exutoire de Camon, de l'efficacité de cet exutoire et du taux de mortalité consécutif au passage des poissons par les turbines de l'usine de Pointis. L'efficacité de l'exutoire de Camon a été estimée à partir des tests effectués lors de la même période (CROZE, CHANSEAU et LARINIER, 1999). La mortalité des smolts transitant par les turbines de Pointis a été évaluée à partir des résultats des essais réalisés sur place par LAUTERS et JUBERT (1996).

\section{RÉSULTATS}

\section{Comportement des smolts en amont de la prise d'eau}

\section{Déplacements des smolts du lâcher à l'arrivée dans le secteur d'étude}

Un seul smolt radiomarqué n'a pas dévalé dans les minutes qui ont suivi son lâcher. Il a stationné pendant 11 jours dans une zone de $250 \mathrm{~m}$ située en aval immédiat de la prise d'eau du canal d'amenée. Les 27 autres smolts radiomarqués ont mis des temps relativement faibles pour parcourir les $685 \mathrm{~m}$ séparant le lieu de lâcher de la zone étudiée : moins de 10 minutes à plus de 21 minutes ont été nécessaires à ces poissons pour arriver à proximité des grilles de l'usine, le délai moyen étant de 13,5 minutes ce qui correspond à une vitesse moyenne de déplacement de $3,2 \mathrm{~km} / \mathrm{h}$.

Les vitesses de dévalaison des smolts dans le canal d'amenée de Pointis sont corrélées aux vitesses moyennes de l'écoulement $\left(p<0,0001 ; r^{2}=0,47\right)$ (Figure 6). En excluant le smolt resté pendant 11 jours aux environs du point de lâcher, ces vitesses de dévalaison ont varié de $0,54 \mathrm{~m} / \mathrm{s}$ à $1,2 \mathrm{~m} / \mathrm{s}$ alors que les vitesses débitantes dans le canal d'amenée (rapport du débit sur la section moyenne de l'écoulement) ont évolué entre 0,48 et $0,81 \mathrm{~m} / \mathrm{s}$, plus de $90 \%$ des smolts dévalant à des vitesses supérieures de $0,1 \mathrm{~m} / \mathrm{s}$ à $0,4 \mathrm{~m} / \mathrm{s}$ à celles de l'écoulement. 


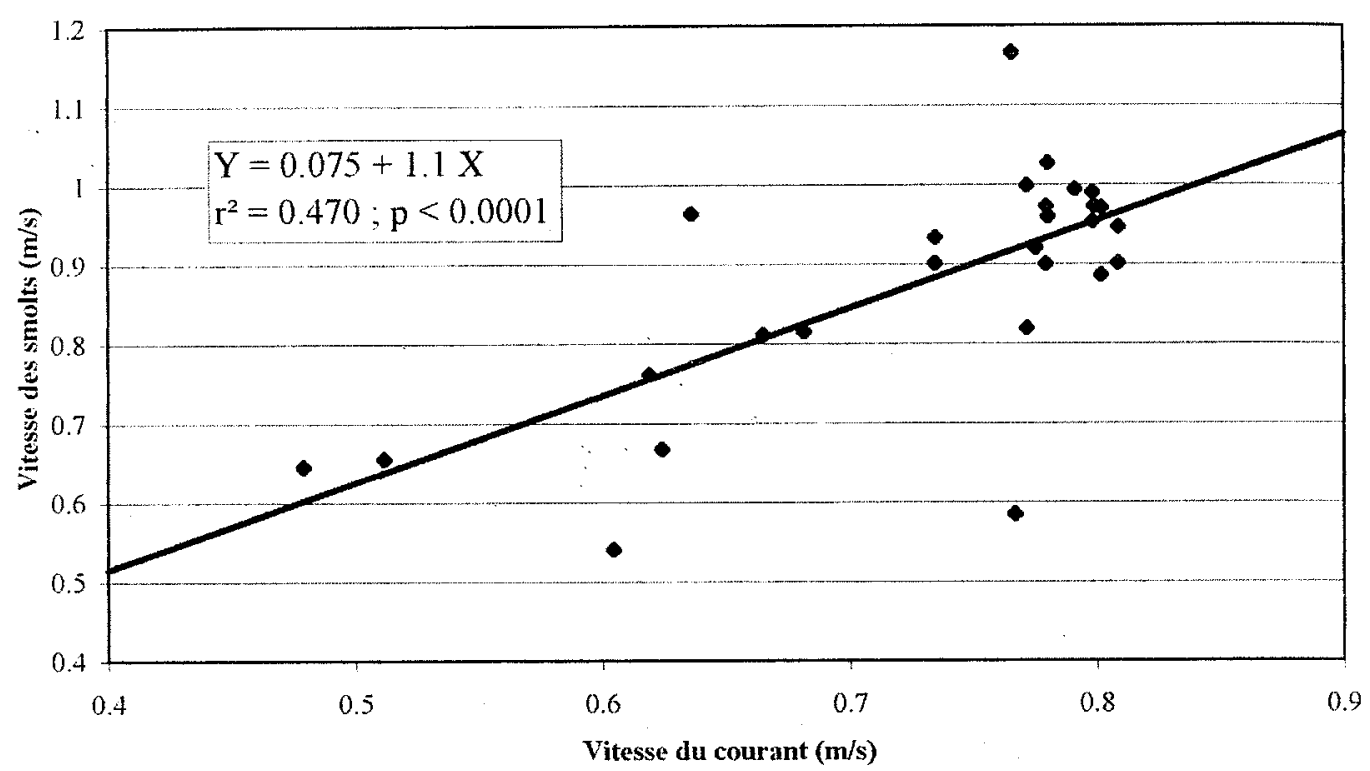

Figure 6

Relation entre la vitesse des smolts dans le canal d'amenée de Pointis et la vitesse du courant.

\section{Figure 6}

Relationship between downstream migration velocity of smolts in the intake canal of Pointis and flow velocity.

\section{Temps de présence en amont des grilles}

Deux groupes de poissons ont pu être distingués. Le premier correspond à des smolts, au nombre de 5 (soit $18 \%$ des poissons radiomarqués), qui franchissent très rapidement les grilles, en moins de 3,5 minutes.

Tous les autres smolts (23 individus) ont séjourné plus de 9 minutes en amont de la prise d'eau de l'usine. Parmi ces poissons, 8 ont été présents moins de 6 heures, 7 de 6 heures à 2 jours, 5 de 2 à 5 jours et 3 de 5 à plus de 11 jours. La durée médiane de présence en amont des grilles est de 1 jour 4 heures pour une moyenne de plus de 2 jours 3 heures.

\section{Comportement des smolts à l'amont des grilles}

Le comportement des smolts n'est décrit que pour les individus restant plus de 9 minutes en amont de la prise d'eau.

Les durées de présence de ces poissons dans les différentes zones de réception sont inégales. La zone la plus longuement fréquentée est la zone 19, c'est-à-dire la zone la plus proche de la rive gauche et située à l'amont immédiat des grilles : les smolts ont passé en moyenne $25 \%$ de leur durée totale de présence sur le site dans cette zone. Les autres zones de stationnement préférentielles sont les zones 13 (10\% du temps total de présence), 14 ( $9 \%$ du temps total de présence) et 17 ( $9 \%$ du temps total de présence).

Les smolts passent $61 \%$ de leur temps dans les zones situées devant le plan des grilles (zones $14,15,16,17,18$ ou 19). En ne considérant que les temps de présence des poissons dans ces zones, on constate que les smolts fréquentent majoritairement les zones situées en berge ( $67 \%$ du temps de présence).

Même si, à l'amont immédiat de la prise d'eau, certains parcours ont été répétés à plusieurs reprises, aucun trajet type n'a été mis en évidence : les déplacements des poissons sont très variables et s'effectuent sur toute la zone d'étude. 
Dix-neuf de ces smolts radiomarqués ont effectué au moins une traversée du canal d'amenée en longeant les grilles de la prise d'eau de l'usine ; 13 de ces 19 individus ont réalisé plus de 10 de ces traversées. Certains poissons ont même longé le plan de grille plusieurs centaines de fois. Ces passages devant les grilles sont plus nombreux dans le sens rive gauche-rive droite (Wilcoxon, $Z=2,34 ; p=0,0191$ ), ce qui est certainement lié à la présence, sur quelques dizaines de centimètres en amont des grilles, d'un faible courant transversal dirigé vers la rive droite.

\section{Franchissement des grilles}

Cinquante-six pour cent des smolts radiomarqués ont franchi l'aménagement de Pointis après avoir traversé le canal d'amenée dans toute sa largeur en amont immédiat des grilles. Les deux tiers de ces traversées ont été effectuées de la rive gauche vers la ive droite contre un tiers dans le sens opposé.

Les zones préférentielles de franchissement des grilles sont celles situées à proximité des berges (zones 15 et 19), avec respectivement 10 individus en rive droite et 8 en rive gauche. Six individus empruntent la zone 16, 2 la zone 18 et un seul le milieu de grille.

\section{Influence du nycthémère sur les déplacements des poissons}

La moitié des 28 poissons radiomarqués a dévalé l'aménagement de Pointis la nuit même du lâcher. Les 14 autres smolts ont franchi les grilles de l'usine au plus tôt la nuit suivante. Parmi ces 14 individus, 11 ont dévalé de nuit, les 3 autres smolts franchissant les grilles pendant la journée. Au total, $89 \%$ des poissons radiomarqués sont passés à l'aval de l'aménagement pendant la nuit.

Les nombres de changements de zone de réception à l'amont immédiat des grilles de prise d'eau (zones 13 à 19) réalisés par les individus présents sur le site la journée ne sont pas significativement différents de ceux réalisés par les individus présents les nuits sans éclairage (ANOVA, $F=0,88 ; p=0,36$ ). Cependant, certains de ces poissons se sont déplacés dans un large secteur englobant la totalité de la zone d'étude en journée alors qu'ils ont fréquenté principalement l'amont immédiat des grilles pendant les nuits sans éclairage (Figure 7).

\section{Influence d'un éclairage nocturne}

Parmi les 28 smolts radiomarqués, 11 ont été présents sur le site au cours de nuits avec éclairage, 13 pendant des nuits sans éclairage et 4 ont été soumis aux deux conditions d'éclairage.

Les durées de présence de ces 4 derniers smolts dans chaque zone de réception lors des deux conditions d'éclairage ne sont pas identiques (Wilcoxon, $Z=2,04 ; p<0,05$ ). Ces individus évoluent principalement entre la zone située sous la lampe (zone 19) et celle située juste en amont (zone 13) lorsque la lampe est allumée alors qu'ils effectuent des parcours plus longs et plus variés lorsque la lampe est éteinte (Figure 8).

Les 15 smolts présents en période d'éclairage restent en moyenne significativement plus de temps en rive gauche qu'en rive droite (Kruskal-Wallis, $p<0,05$ ) (Figure 9) : ils stationnent en moyenne $67 \%$ du temps passé devant les grilles en zone 19 contre seulement $12 \%$ en zone " $14+15$ " (équivalent à la zone 19 en rive droite). Par contre, pour les 17 smolts présents les nuits sans éclairage, aucune préférence pour l'une des deux rives n'apparaît (Kruskal-Wallis, $p=0,58$ ). 


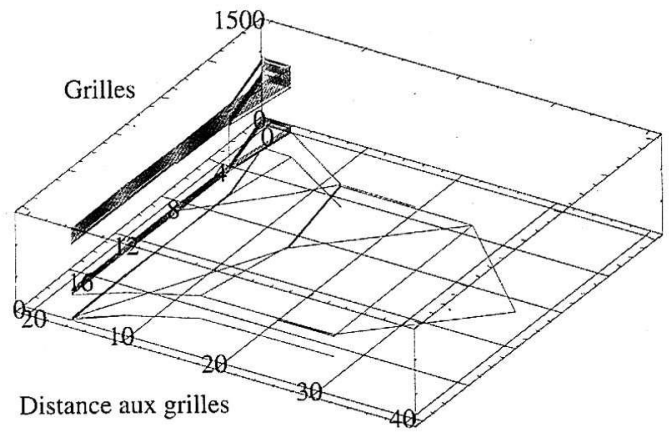

Déplacements du poisson 48821 de nuit (sans éclairage)

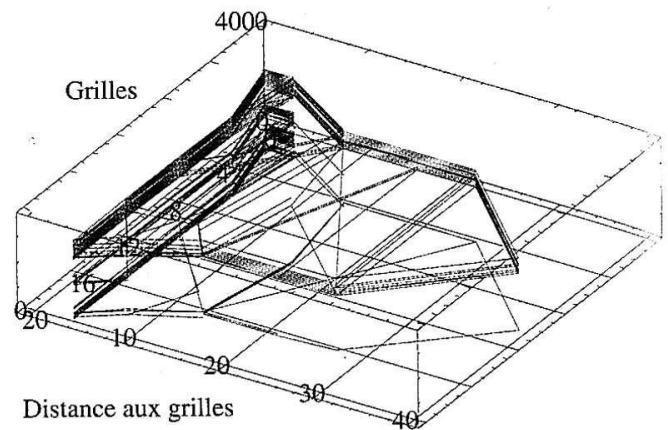

Déplacement du poisson 48821 de jour

\section{Figure 7}

Exemple de graphe de déplacements d'un smolt radiomarqué de jour et de nuit sans éclairage.

\section{Figure 7}

Diagram showing radiotagged smolt displacements during day and during night without lighting.

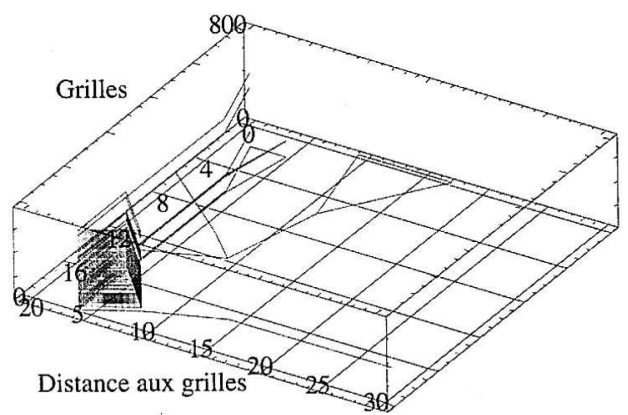

Déplacement du poisson $48782 \mathrm{~A}$ de nuit (avec éclairage)

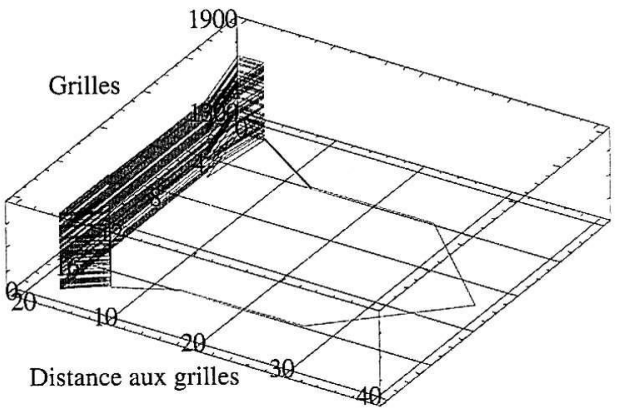

Déplacement du poisson 48782A de nuit (sans éclairage)

\section{Figure 8}

Exemple de graphe de déplacements nocturnes d'un smolt radiomarqué avec et sans éclairage.

\section{Figure 8}

Diagram showing radiotagged smolt nocturnal displacements with and without lighting. 
Pourcentage de durée de présence devant les grilles lors des nuits avec lampe

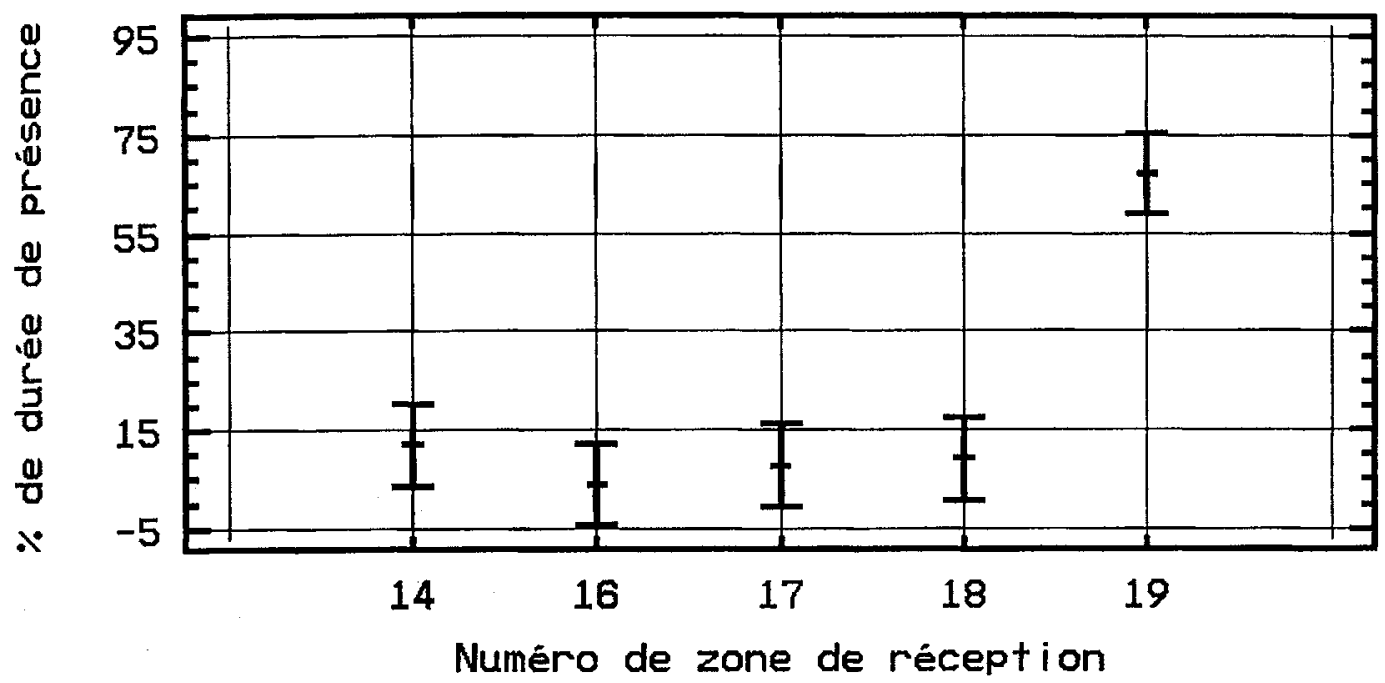

Pourcentage de durée de présence devant les grilles lors des nuits sans lampe

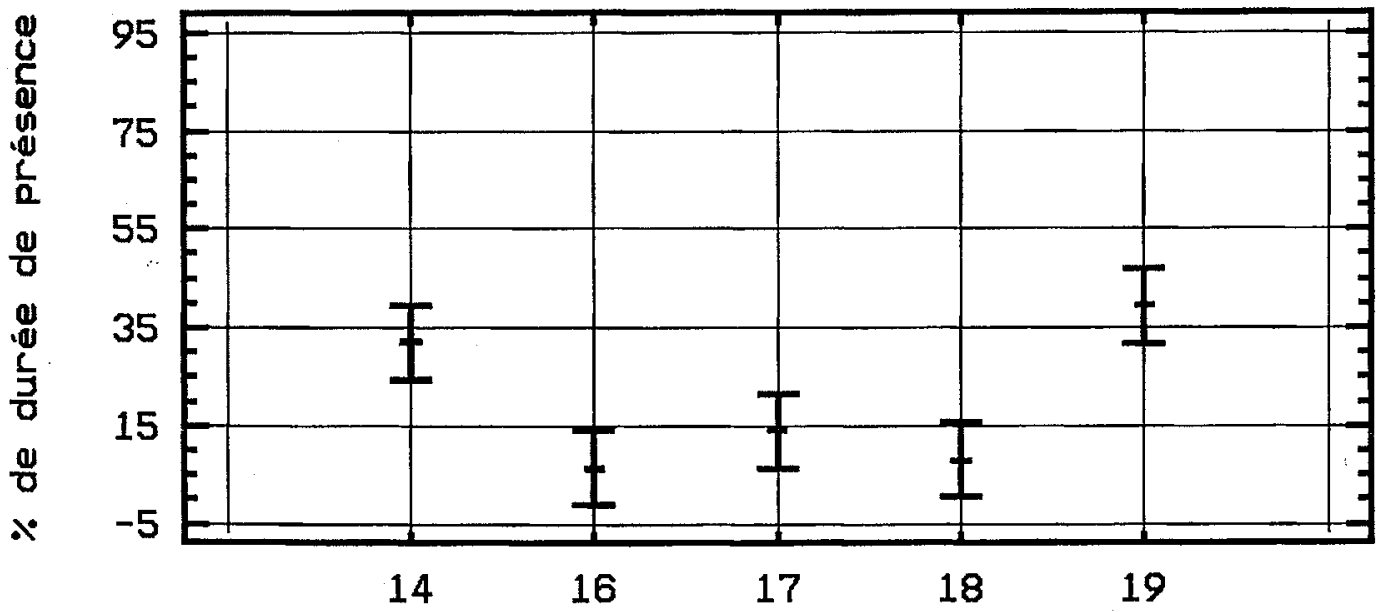

Numéro de zone de réception

Figure 9

Comparaison des pourcentages de durée de présence nocturne des smolts radiomarqués au niveau des zones situées devant les grilles avec et sans éclairage.

Figure 9

Comparison of nocturnal presence time percentages of radiotagged smolts in areas in front of trashrack with and without mercury light. 
La répartition des zones de franchissement des grilles lors du passage à l'aval $\mathbf{n}$ diffère pas significativement entre les nuits avec éclairage et celles sans éclairag: (Wilcoxon, $p=0,79$ ).

\section{Estimation des dévalaisons par le barrage de Rodère}

Lorsque le débit de la Garonne devient supérieur au débit prélevé par le canc: d'amenée de l'usine de Camon, un déversement se produit au barrage de Rodère et les: smolts lâchés en amont de. l'usine de Pointis ont alors la possibilité de transiter par le; ouvrages évacuateurs du barrage.

Sur les 406 smolts lâchés en amont de Pointis, 226 ont transité par l'exutoire d: Camon, ce qui correspond à un pourcentage de recapture de $56 \%$ (Tableau I). C: pourcentage est cependant très variable selon les lots (de $14 \%$ à $79 \%$ ).

\section{Tableau I}

Récapitulatif des recaptures dans le piège de Camon des différents lots dê smolts lâchés à Pointis.

\section{Table I}

Summary of recaptures in the Camon trap of the batches of smolts released upstream from Pointis.

\begin{tabular}{|c|c|c|c|c|}
\hline $\begin{array}{l}\text { Date et heure } \\
\text { du lâcher }\end{array}$ & $\begin{array}{l}\text { Nombre } \\
\text { de smolts } \\
\text { lâchés }\end{array}$ & $\begin{array}{c}\text { Nombre } \\
\text { de smolts recapturés } \\
\text { à Camon }\end{array}$ & $\begin{array}{c}\text { Pourcentage } \\
\text { de smolts recapturés } \\
\text { à Camon }\end{array}$ & $\begin{array}{c}\text { Surverse moyenne } \\
\text { à Rodère } \\
\left(\mathrm{m}^{3} / \mathrm{s}\right)\end{array}$ \\
\hline 06/04 à 23:37 & 22 & 16 & $73 \%$ & 3 \\
\hline 08/04 à 22:56 & 20 & 13 & $65 \%$ & 2 \\
\hline 14/04 à 22:05 & 21 & 15 & $71 \%$ & 8 \\
\hline $15 / 04$ à $22: 18$ & 21 & 16 & $76 \%$ & 8 \\
\hline 22/04 à 00:06 & 22 & 13 & $59 \%$ & 16 \\
\hline $22 / 04$ à $22: 48$ & 22 & 16 & $73 \%$ & 14 \\
\hline 24/04 à 00:13 & 21 & 13 & $62 \%$ & 14 \\
\hline 27/04 à 22:41 & 22 & 4 & $18 \%$ & 73 \\
\hline 28/04 à 23:21 & 22 & 11 & $50 \%$ & 43 \\
\hline $29 / 04$ à $22: 48$ & 21 & 11 & $52 \%$ & 26 \\
\hline $30 / 04$ à 23:30 & 22 & 16 & $73 \%$ & 26 \\
\hline 04/05 à 22:59 & 22 & 7 & $32 \%$ & 25 \\
\hline 06/05 à 00:08 & 22 & 14 & $64 \%$ & 25 \\
\hline 06/05 à 23:42 & 21 & 15 & $71 \%$ & 18 \\
\hline 08/05 à 00:05 & 19 & 15 & $79 \%$ & 18 \\
\hline 11/05 à 22:36 & 21 & 10 & $48 \%$ & 30 \\
\hline 13/05 à 22:27 & 22 & 10 & $45 \%$ & 44 \\
\hline \multirow[t]{2}{*}{ 15/05 à $21: 57$} & 22 & 3 & $14 \%$ & 57 \\
\hline & 21 & 8 & $38 \%$ & 36 \\
\hline Total & 406 & 226 & $56 \%$ & \\
\hline
\end{tabular}


Cette grande variabilité peut être attribuée aux déversements au barrage de Rodère. On a porté sur la Figure 10 l'évaluation de la proportion de smolts dévalant par le barrage de Rodère en fonction de la proportion du débit total de la Garonne déversant par le barrage. Pour des débits déversés de $20 \%$ à $30 \%$, le pourcentage de dévalaison au barrage n'est que de $10 \%$ à $20 \%$. Il faut attendre des débits déversés correspondant à $40-50 \%$ du débit de la Garonne pour voir dévaler par le barrage $70 \%$ des poissons.

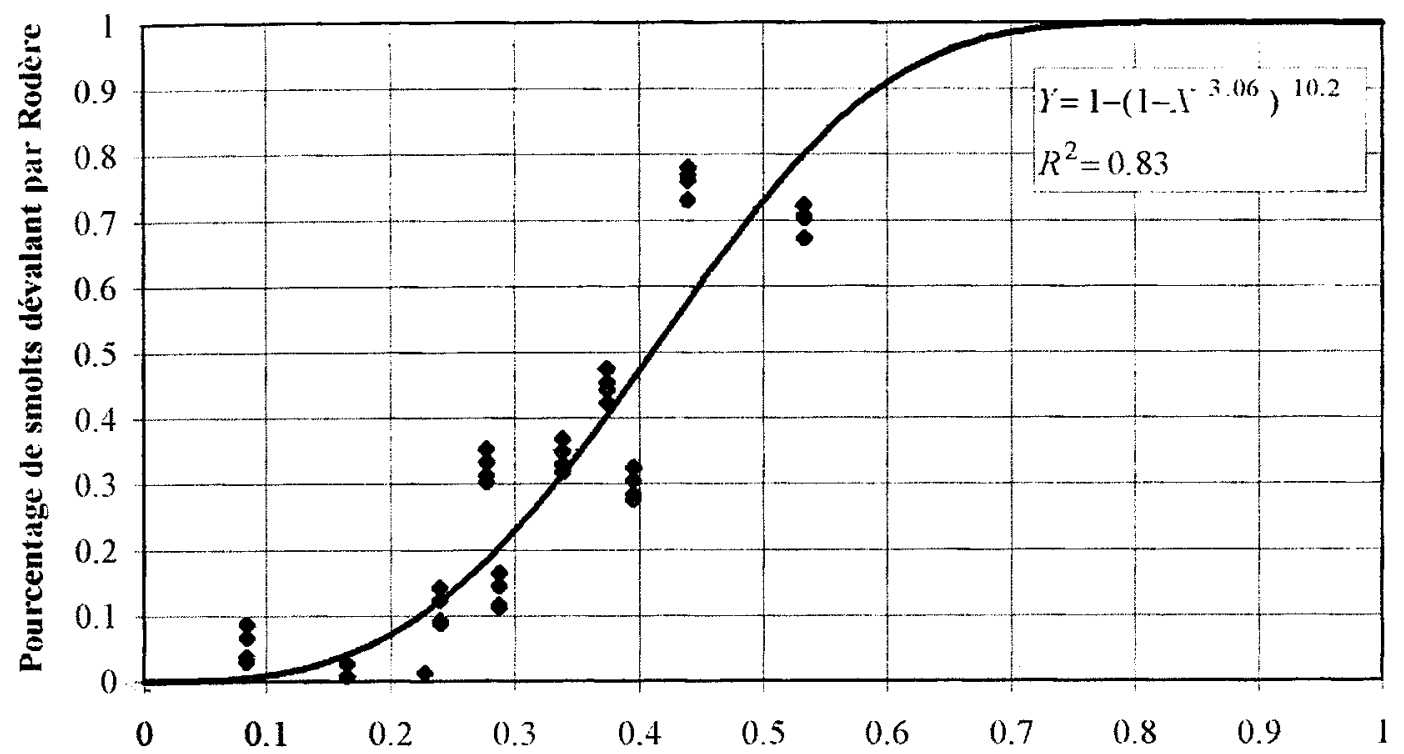

Débit déversé à Rodère / débit total de la Garonne

Figure 10

Estimation du pourcentage de smolts dévalant par le barrage de Rodère en fonction du rapport « débit déversant au barrage de Rodère / débit total de la Garonne ».

\section{Figure 10}

Estimate of smolt percentage passing downstream from the Rodère dam as a function of the ratio «spilling discharge at Rodèrə dam / total Garonne flow ».

\section{Durées des trajets de Pointis à Camon}

Près de la moitié (48\%) des smolts marqués à l'aide de transpondeurs mettent moins de 24 heures après avoir été lâchés en amont de Pointis pour rejoindre le piège de Camon. Le délai moyen pour parcourir cette distance de $5,4 \mathrm{~km}$ est de 1 jour 20 heures. Le smolt le plus rapide a atteint Camon en 1 heure 17 minutes, le plus lent a réalisé le même parcours en 12 jours.

Les délais de recapture semblent varier selon les débits de la Garonne (ANOVA, $F=27,92 ; p<0,001)$. Il apparaît en effet que le temps nécessaire aux smolts lâchés à Pointis pour rejoindre Camon est en moyenne de moins de 23 heures lorsque le débit de la Garonne est supérieur à $90 \mathrm{~m}^{3} / \mathrm{s}$ contre près de 2 jours 12 heures en moyenne pour des débits inférieurs.

Les smolts radiomarqués ont aussi été suivis après leur passage à travers les turbines de l'usine de Pointis. Le temps nécessaire à ces poissons pour effectuer les $1,25 \mathrm{~km}$ séparant l'aval de Pointis du barrage de Rodère est connu pour 13 individus. II varie de 22 minutes à plus de 1 jour ce qui correspond à des vitesses variant de $3,4 \mathrm{~km} / \mathrm{h}$ à $0,05 \mathrm{~km} / \mathrm{h}$. La valeur médiane du temps nécessaire à ce parcours est de 33 minutes, soit $2,3 \mathrm{~km} / \mathrm{h}$. 
Le suivi de 10 de ces smolts radiomarqués du barrage de Rodère jusqu'à la prise d'eau de l'usine de Camon, sur un linéaire de $3,4 \mathrm{~km}$ de canal d'amenée, indique de: vitesses de déplacement largement supérieures à celles observées sur le tronço: précédent. Cette distance est en effet parcourue en des temps variant de 29 minutes a 1 heure 22 minutes (vitesse variant de $7 \mathrm{~km} / \mathrm{h}$ à $2,5 \mathrm{~km} / \mathrm{h}$ ) pour une valeur médiane de 32 minutes (vitesse de $6,3 \mathrm{~km} / \mathrm{h}$ ). Les vitesses de dévalaison des smolts dans le cancil d'amenée de Camon sont corrélées aux vitesses débitantes de l'écoulement dans le cane. $\left(p=0,025 ; r^{2}=0,49\right)$ (Figure 11). Ces vitesses de dévalaison ont varié de $0,68 \mathrm{~m} / \mathrm{s}$ at $1,9 \mathrm{~m} / \mathrm{s}$ alors que celles de l'écoulement ont évolué entre $0,93 \mathrm{~m} / \mathrm{s}$ à $1,4 \mathrm{~m} / \mathrm{s}$, les vitesse's des smolts étant supérieures de $0,2 \mathrm{~m} / \mathrm{s}$ à $0,7 \mathrm{~m} / \mathrm{s}$ à celles de l'écoulement.

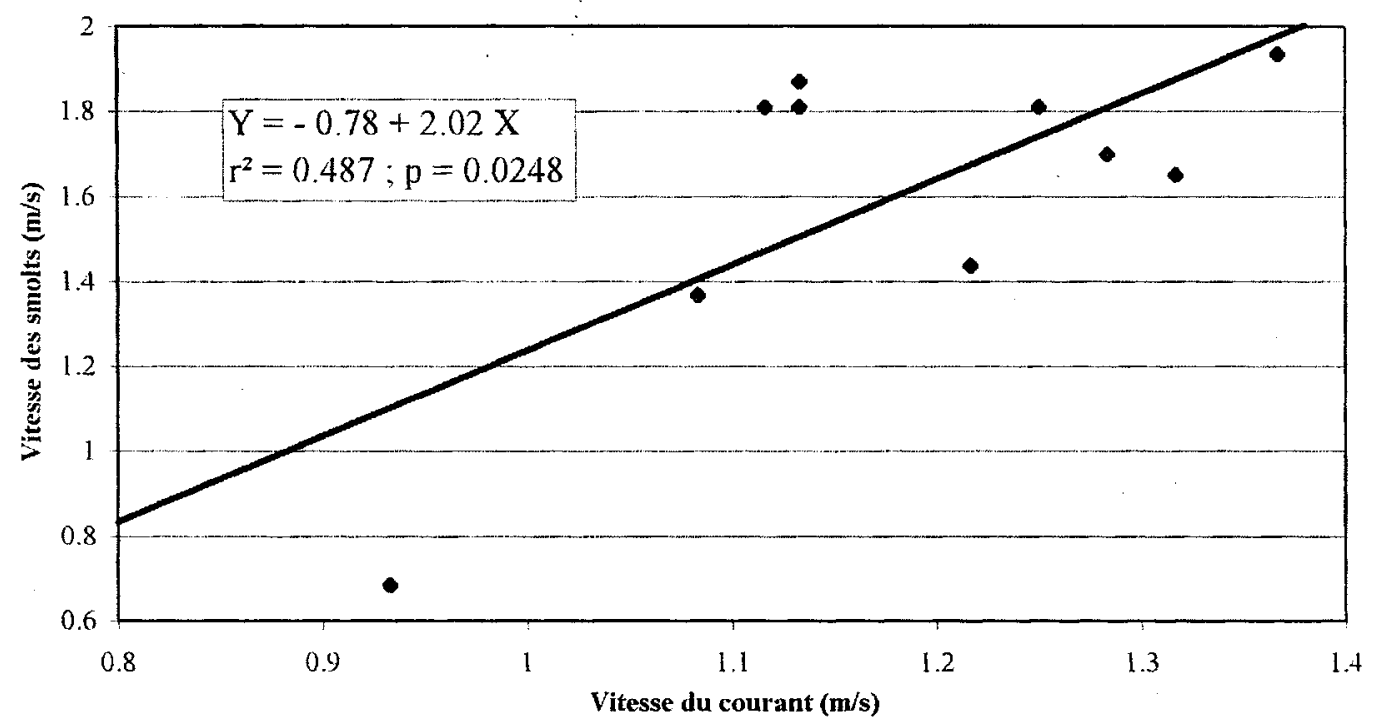

Figure 11

Relation entre la vitesse des smolts dans le canal d'amenée de Camon et la vitesse du courant.

Figure 11

Relationship between downstream migration velocity of smolts in the intake canal of Camon and flow velocity.

\section{DISCUSSION}

L'étude menée au niveau de la prise d'eau de l'usine hydroélectrique de Pointis avait comme premier objectif de suivre les déplacements de smolts de saumon atlantique radiomarqués de façon à déterminer l'emplacement d'un exutoire de dévalaison.

De même qu'au niveau de la prise d'eau de Bedous où $11 \%$ à $14 \%$ des smolts franchissaient directement les grilles (CHANSEAU, LARINIER et TRAVADE, 1999), un pourcentage non négligeable des smolts $(18 \%)$ ne reste que très peu de temps en amont de la prise d'eau de Pointis. Ces poissons n'ont donc que très peu de chance de trouver l'entrée d'un exutoire. Un tel comportement met en évidence une des limites des exutoires de dévalaison associés aux grilles de prise d'eau conventionnelles, notamment dans le cas de Pointis où l'espacement entre les barreaux est important $(5 \mathrm{~cm})$.

Les durées de présence en amont de la prise d'eau des smolts ne franchissant pas directement les grilles sont très largement supérieures (médiane de 1 jour 4 heures) à celles observées sur le site de Bedous (médiane de 34 minutes en 1995 et de 1 heure en 1998) (CHANSEAU, LARINIER et TRAVADE, 1999) ou sur le site de Camon (médiane de 9 minutes) (CROZE, CHANSEAU et LARINIER, 1999). Ces durées importantes, qui 
peuvent paraître relativement surprenantes compte tenu de l'espacement entre les barreaux des grilles de la prise d'eau $(5 \mathrm{~cm})$, s'expliquent par les faibles vitesses d'écoulement à l'approche du plan de grille $(<0,5-0,6 \mathrm{~m} / \mathrm{s})$. De telles conditions hydrauliques ont par ailleurs permis au poisson d'explorer la totalité de la prise d'eau, à la différence des sites de Bedous et de Camon, où les vitesses dans le canal d'amenée étaient plus importantes.

Suite à l'installation d'une lampe à vapeur de mercure en rive gauche, il est apparu que les smolts radiomarqués étaient rencontrés significativement plus souvent sous la lampe lorsque celle-ci était allumée. Ces résultats, qui mettent en évidence les effets attractifs d'un éclairage nocturne sur les déplacements des smolts, sont comparables à ceux obtenus notamment par PUCKETT et ANDERSON (1988), TAFT (1988), LARINIER et BOYER-BERNARD (1991) et EPRI (1994).

Une relation a été obtenue entre la proportion de smolts dévalant par le barrage de Rodère et la part du débit total de la Garonne déversant au niveau de ce barrage. Avec des débits déversés de l'ordre du $1 / 10$ du débit du cours d'eau, cette relation fait apparaître l'absence de passage par les ouvrages évacuateurs. II faut attendre des déversements de l'ordre de $70 \%$ du débit total du cours d'eau pour voir la quasi-totalité des poissons transiter par le barrage. Ce résultat confirme la nécessité de l'installation d'un dispositif de dévalaison au niveau de la prise d'eau de l'usine de Camon, des déversements correspondant au débit réservé n'étant pas suffisants pour éviter l'entraînement des smolts dans le canal d'amenée.

Dans le canal d'amenée de Pointis comme dans celui de Camon, la vitesse de dévalaison des smolts est corrélée à la vitesse moyenne de l'écoulement, les poissons dévalant d'autant plus rapidement que les vitesses de l'écoulement sont importantes. Cette vitesse de dévalaison est généralement légèrement supérieure à la vitesse débitante moyenne de l'écoulement (rapport du débit sur la section mouillée moyenne du canal). Une des explications les plus probables est que, comme l'ont observé plusieurs auteurs (HANSEN et JONSSON, 1985 ; BAKSHTANSKY, NESTEROV et NEKLYUDOV, 1987) les smolts dévalent préférentiellement dans les zones les plus vives de l'écoulement, en particulier dans la couche de surface, où les vitesses sont plus élevées que la vitesse débitante.

\section{CONCLUSION}

L'intérêt de cette expérimentation a été de montrer, sur un site non équipé d'exutoire de dévalaison, où les vitesses dans le canal d'amenée sont relativement faibles, que les smolts de saumon atlantique en dévalaison pouvaient rester jusqu'à plusieurs jours en amont des grilles de la prise d'eau. Pendant cette durée, les poissons ont présenté un comportement exploratoire marqué, la plupart d'entre eux se déplaçant sur l'ensemble du site d'étude. Dans de telles conditions, on peut penser qu'un dispositif de dévalaison installé au niveau de l'une des deux rives devrait se révéler relativement efficace, même s'il est à prévoir que cette efficacité ne serait que partielle, dans la mesure où déjà près de $20 \%$ des individus franchissent très rapidement le plan de grilles. Ce pourcentage élevé est vraisemblablement lié à l'espacement important des grilles.

Cette étude a mis une nouvelle fois en évidence l'importance des conditions hydrodynamiques sur le comportement de dévalaison. Si les méthodes de radiotélémétrie permettent d'assez bien appréhender le comportement du poisson, il reste à développer et mettre en oeuvre des outils (courantomètres Doppler, traçeurs...) permettant de mieux caractériser les écoulements, que ce soit au niveau des prises d'eau ou dans les canaux d'amenée, afin d'étudier plus finement les réponses des poissons à un champ de vitesses donné. 


\section{REMERCIEMENTS}

Cette étude a pu être menée à bien grâce à l'implication sur le terrain de $\mathrm{S}$. BOSC L. CARRY et A. LALLEMAND. Nous remercions, la FDPPMA de la Haute-Garonne pou: avoir mis à notre disposition des bassins de la pisciculture de Soueich ainsi qu'EDF, GEF de Saint-Gaudens, et plus particulièrement l'ensemble du personnel de l'usine de Camon EDF et MI.GA.DO. ont assuré respectivement les maîtrises d'ouvrage et d'oeuvre df l'étude financée dans le cadre de la Convention Garonne.

\section{BIBLIOGRAPHIE}

BAGLINIERE J.L., 1976. Etude des populations de saumon atlantique (Salmo salar L. 1766) en Bretagne-Basse-Normandie. 1 - Caractéristiques des smolts de la rivière Ellé. Ann. Hydrobiol., 7, 141-158.

BAKSHTANSKY E.L., NESTEROV V.D., NEKLYUDOV M.N., 1987. Development of schooling behaviour in juvenile Atlantic salmon, Salmo salar, during seaward migration. Vopr. ikhtiologii, 6, 1000-1009.

BOUSQUET B., 1979. Biologie et migration des smolts de saumon atlantique (Salmo salar L. 1766) dans les bassins Loire-Allier et Adour-Gave d'Oloron. Thèse de Doctorat Inst. Nat. Polytech., Toulouse, France, 67 p.

CHANSEAU M., LARINIER M., TRAVADE F., 1999. Efficacité d'un exutoire de dévalaison pour smolts de saumon atlantique (Salmo salar L.) et comportement des poissons au niveau de l'aménagement hydroélectrique de Bedous sur le Gave d'Aspe étudiés par la technique de marquage-recapture et par radiotélémétrie. Bull. Fr. Pêche Piscic., 353/354, 99-120.

CROZE O., CHANSEAU M., LARINIER M., 1999. Efficacité d'un exutoire de dévalaison pour smolts de saumon atlantique (Salmo salar L.) et comportement des poissons au niveau de l'aménagement hydroélectrique de Camon sur la Garonne. Bull. Fr. Pêche Piscic., 353/354, 121-140.

EPRI, 1994. Research update on fish protection technologies for water intakes. EPRI, TR-104122, $208 \mathrm{p}$.

HANSEN L.P., JONSSON B., 1985. Downstream migration of hatchery-reared smolts of Atlantic salmon (Salmo salar L.) in the River Imsa, Norway. Aquaculture, 45, 237-248.

LARINIER M., BOYER-BERNARD S., 1991. Dévalaison des smolts et efficacité d'un exutoire de dévalaison à l'usine hydroélectrique d'Halsou sur la Nive. Bull. Fr. Pêche Piscic., 321, 72-92.

LAUTERS F., JUBERT F., 1996. Estimation de la mortalité des smolts de saumons atlantiques lors du passage dans les turbines des usines du groupement de Camon. EDF-DTG, D 4160 / DTG / RE-ENV / 96-113-A, 28 p.

METCALFE N.B., THORPE J.E., 1990. Determinants of geographical variation in the age of seaward-migrating salmon, Salmo salar. J. Anim. Ecol., 59, 135-145.

PUCKETT K.J., ANDERSON J.J., 1988. Behavioral responses of juvenile salmonids to strobe and mercury lights. Univ. of Washington, Fish. Res. Inst., Tech. Rep. FRI-UW-8717, $35 \mathrm{p}$.

TAFT N., 1988. Evaluations of fish protection systems for use at hydroelectric plants. Hydro Review, 54-62. 